\title{
Photocatalytic degradation of malachite green dye using zero valent iron doped polypyrrole
}

\author{
Bircan HASPULAT TAYMAZ ${ }^{1+}$, Handan KAMIŞ ${ }^{1}$, Özge YOLDAŞ ${ }^{2}$ \\ ${ }^{1}$ Konya Technical University, Chemical Engineering Department, Konya, Turkey \\ ${ }^{2}$ Lucoil Lubricants Middle East Madeni Yağlar San ve Tic A.Ş., İmir, Turkey
}

\begin{abstract}
The zero valent iron doped polypyrrole (Ppy/Fe) was synthesized via chemical oxidative polymerization method in diethylene glycol medium for the first time in this study. The characterization of synthesized Ppy and Ppy/Fe composites was realized by using fourier transform infrared spectrometer (FTIR), UV-visible diffuse reflectance spectrometer (DRS), scanning electron microscope (SEM), elemental dispersion X-ray analysis (EDX), four point probe electrical conductivity X-Ray diffraction spectroscopy (XRD) and $\mathrm{pH}$ at point of zero charge (pHpzc) methods. These characterizations show that there is an interaction between zero valent iron $\left(\mathrm{Fe}^{0}\right)$ and Ppy polymer. The photocatalytic activity of synthesized Ppy and Ppy/Fe composite were investigated by degradation of malachite green (MG) dye under UV light irradiation. Ppy/Fe composite was achieved complete degradation within $60 \mathrm{~min}$, which is 5.92 times faster than pure Ppy. The effect of irradiation time, ratio of Ppy and $\mathrm{Fe}^{0}$ in the synthesized composites, photocatalyst amount to photocatalytic efficiency of Ppy/Fe is studied. Also, the photocatalytic stability of Ppy/Fe is investigated under UV light illumination for degradation MG dye.
\end{abstract}

Keywords: Chemical oxidative polymerization, Polypyrrole, Ppy/Fe, Photocatalysis, Malachite green

\section{Introduction}

In many developing countries, textile industries are essential for economic development. Different materials like cotton, fibers, and chemicals, including dyes, have been used in textile industries [1]. These synthetic dyes are harmful, detrimental to mammals, and aquatic environments. Due to the infertile dyeing process used in textile industries, huge synthetic dyes are charged into the environment and about $17-20 \%$ of industrial wastewater is produced from the textile dyeing and finishing process [2]. Although these industries ensure critical economic advantages, they also pollute the environment to generate toxic wastewater. An effective treatment method should be applied before it is dumped into the environment to reduce the negative effects of textile wastewater. Textile wastewater has been treated using various technologies like adsorption, membrane filtration, coagulation, ion exchange, biological treatment, photocatalysis, etc. Among these treatment methods, photocatalysis has attracted a great deal in recent years [3, 4]. In a photocatalytic reaction, the photon energy, hv, is equal or exceeds the band gap of the semiconductor; a photoexcited electron (e-) is jumped from the valance band (VB) of a photocatalyst to the conduction band (CB) [5-7]. So, generated e-/h+ couples transferred to the semiconductor's surface and then react with the other reactants on the photocatalyst surface [8]. An ideal photocatalyst has a suitable band gap, large surface area, and low cost [9]. In recent years, conductive polymers like polypyrrole (Ppy), polyaniline, and polythiophene are getting attention due to its easy synthesis procedure, chemical stability, low cost and good conductivity photocatalysis applications. Ppy is a highly preferred conductive polymer due to conjugated structure; Ppy has good electron transport properties with excellent photoinduced charge separation [10]. Ppy has been synthesized easily using chemical $[11,12]$ and electrochemical [13] methods. In the literature, Ppy composite polymer photocatalysts have been used for the photocatalytically organic dye degradation process [14-16]. Zero valent iron $\left(\mathrm{Fe}^{0}\right)$ due to its small particle size and reduction properties used as a catalyst in wastewater treatment. $\mathrm{Fe}^{0} \mathrm{NPs}$ have superparamagnetic properties, non-toxic, low cost, and easy synthesis procedure. Although these unique properties, magnetic Fe0 tends to agglomeration due to high surface energy and superparamagnetic properties in waste-
This is an Open Access article distributed under the terms of the Creative Commons Attribution Non-Commercial License (http://creativecommons.org/licenses/by-nc/3.0/) which permits unrestricted non-commercial use, distribution, and reproduction in any medium, provided the original work is properly cited.

Copyright (C) 2022 Korean Society of Environmental Engineers
Received November 23, 2020 Accepted February 15, 2021

${ }^{\dagger}$ Corresponding author

E-mail: bhaspulat@ktun.edu.tr

Tel: +903322051717

ORCID: 0000-0002-5170-322X 
water medium [17]. To overcome these drawbacks, magnetic $\mathrm{Fe}^{0}$ NPs combined with various materials like metal oxide [18-20], conductive polymers [21-23].

There are various studies about Ppy/Fe composite in the literature. However, up to our knowledge, no published study described the use of Ppy/Fe composite in the degradation of MG dyes in the wastewater. This study aims to synthesize Ppy/Fe composite with a facile chemical oxidative polymerization method for degradation MG dye. The characterization of Ppy/Fe was completed via FTIR, DRS, SEM, EDX, four probe electrical conductivity, XRD and pHpzc methods. The photocatalytic efficiency of Ppy/Fe composite was investigated under UV light irradiation as a function of irradiation time, the ratio of Ppy and $\mathrm{Fe}^{0}$, photocatalyst amount, and photocatalytic stability.

\section{Experimental}

\subsection{Materials}

Pyrolle (py) monomer was purchased from Merck Chemical and distilled under atmosphere pressure before use. Ammonium peroxidisulphat (APS), diethylene glycol (DEG), Fe(NO3) $)_{3} .9 \mathrm{H} 2 \mathrm{O}$, malachite green (MG) were purchased from Merck Chemicals. All reagents were used without purification. Deionized water was used in experiments.

\subsection{Synthesis of Ppy/Fe Photocatalyst}

Ppy polymer was synthesized in aqueous DEG using APS as an oxidant using chemical oxidation polymerization described elsewhere [24]. $\mathrm{Fe}\left(\mathrm{NO}_{3}\right)_{3} .9 \mathrm{H}_{2} \mathrm{O}(0.1 ; 0.2 ; 0.4$ and $0.8 \mathrm{M})$ dissolved in water (50 mL), and a yellow solution was obtained (1). $\mathrm{NaBH}_{4}$ powder was quickly added to the solution until the yellow color vanished (2) [25]. $50 \mathrm{~mL}$ DEG was added to this solution at a concentration of $1.0 \mathrm{M}$ (3). Simultaneously, 0.4 M APS (50 mL) and $0.4 \mathrm{M}$ py monomer $(50 \mathrm{~mL}$ ) were included. The blend was stirred for $30 \mathrm{~min}$ after aged at room temperature for $30 \mathrm{~min}$. Then precipitate washed, filtered, and dried under vacuum oven at 40 for $24 \mathrm{~h}$. The concentration of $\mathrm{Fe}\left(\mathrm{NO}_{3}\right)_{3} \cdot 9 \mathrm{H}_{2} \mathrm{O}$ was changed to from 0.1 to $0.8 \mathrm{M}$, pyrrole monomer concentration kept constant. The obtained polymer composited symbolized as Ppy/Fe (4:1), c) Ppy/Fe (2:1), d) Ppy/Fe (1:1), e) Ppy/Fe (1:2), respectively. The synthesis procedure is illustrated in Fig. S1.

\subsection{Characterization of Ppy/Fe Photocatalyst}

The characterization of Ppy/Fe was completed by using optic, spectroscopic and morphological methods. The optical property of Ppy/Fe was examined using a UV-visible diffuse reflectance spectrometer (DRS) with HITACHI U3900 instrument. Fourier transform infrared spectra FTIR were recorded at 4,000-500 $\mathrm{cm}^{-1}$ wavenumber range by Perkin Elmer 1725. The scanning electron microscope SEM images were recorded with ZEISS Evo LS 10 SEM. The absorption spectra of dye were recorded using the Ocean Optics HR4000 UV-visible spectrophotometer at photocatalytic activity experiments. The pHpzc of Ppy/Fe was determined by $\mathrm{pH}$ drift method. The crystallinity of Ppy and Ppy/Fe were investigated via Shimadzu XRD-6000 X-ray diffractometer $(\mathrm{Cu} K \alpha$ radiation $(\lambda=0.15418 \mathrm{~nm}$; $20-70^{\circ}$ and $\left.2^{\circ} / \mathrm{min}\right)$.

\subsection{Investigation of Photocatalytic Activity of Ppy/Fe}

The photocatalytic efficiency of Ppy/Fe composite was searched by degradation MG dye under UV light illumination in a quartz tube. Photocatalytic activity experiments were carried in a Luzchem 4V (Luzhem Research Inc. Canada) photo reactor with 8 UVC lamps (emission at $254 \mathrm{~nm}$ ). Photocatalytic experiment details were in our previous research [26]. The photocatalysts $\left(\mathrm{x} \mathrm{mg} / \mathrm{mL} ; \mathrm{x}=0.2,0.4,0.8,1.2\right.$ and 1.6) and $1.0^{*} 10^{-5} \mathrm{M} \mathrm{MG}$ dye solution $(3 \mathrm{~mL})$ added a quartz tube. The adsorption/desorption equilibrium of dye on the Ppy/Fe was stored in dark conditions for $60 \mathrm{~min}$. After, UV light switched on for initiate photocatalytic reaction. For 15 minintervals time, the absorption spectra of MG were recorded with UV-vis absorption spectrophotometer. The degradation efficiency of MG dye was calculated from Eq. (1):

$$
\text { Degradation } \%=\frac{C_{0-} C_{t}}{C_{0}} * 100
$$

Where $\mathrm{C}_{0}$ is the initial concentration of dye and $\mathrm{C}_{t}$ is the concentration of dye at time $t$.

\section{Results and Discussion}

\subsection{Characterization of Ppy/Fe Nanocomposite}

The FTIR spectra investigated to analyze the chemical bond of Ppy and Ppy/Fe composites (Fig. 1). The characteristic FTIR peaks of Ppy were observed in Fig. 1(a). The peak at centered at 1,458 $\mathrm{cm}^{-1}$ is assigned to $\mathrm{C}-\mathrm{N}$ stretching vibration in the pyrrole ring [27]. C-C stretching vibration is observed at $1,544 \mathrm{~cm}^{-1}$ correspond to [28]. The peaks at 1,281 and $754 \mathrm{~cm}^{-1}$ are assigned to $\mathrm{C}-\mathrm{H}$. These peaks confirm the polymerization of pyrrole monomer. The peak at around $565 \mathrm{~cm}^{-1}$ in Fig. 1(b)-(e) can be attributed Fe-O stretches in Ppy/Fe composite [29]. The FTIR peaks of Ppy mention

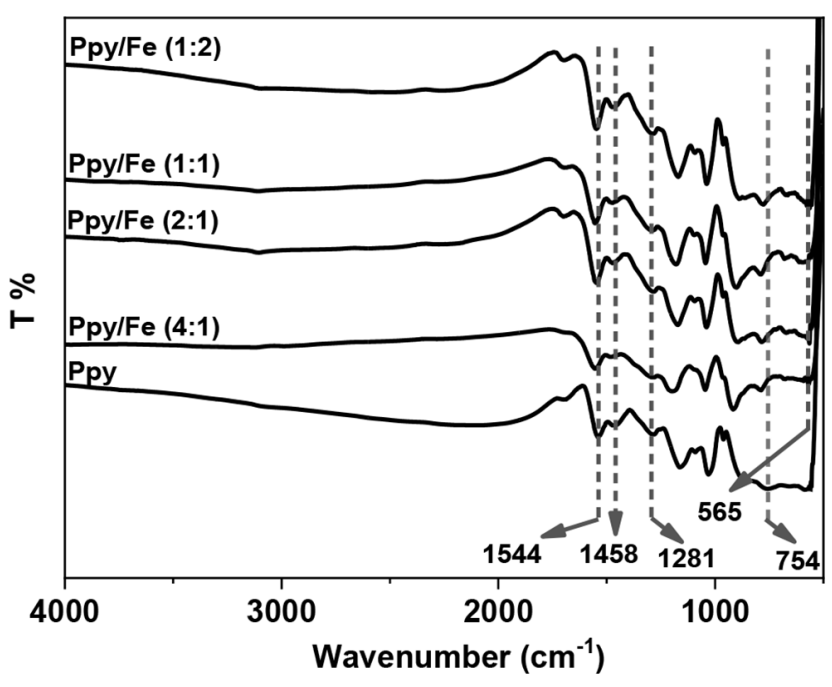

Fig. 1. FTIR spectra of Ppy and Ppy/Fe. 
above partially shifted to higher wavenumber after addition $\mathrm{Fe}^{0}$ particles to polymerization medium due to interaction between Ppy and $\mathrm{Fe}^{0}$.

For investigate the optical properties of Ppy and Ppy/Fe composite, the UV-vis diffuse reflectance spectrum were recorded as given Fig. 2(a). Three possible states of Ppy which are neutral, poloron (radical cation) and bipoloron (dication) can be observed all at the same time. These three characteristic absorption bands of Ppy are seen at $250-270 \mathrm{~nm}, 310-360 \mathrm{~nm}$ and $600-650 \mathrm{~nm}$ [30]. The bands at visible range are attributed to the doping level and introduction of polaron and bipolaron lattices, which represent the protonation stages of Ppy chain [30]. As can be seen from Fig. 3(a) Ppy shows an absorption band at $250 \mathrm{~nm}$ and a shoulder at around $310 \mathrm{~nm}$. The absorption peak at $210 \mathrm{~nm}$ which is character- istic $\pi-\pi^{*}$ transition of neutral Ppy [31]. With the addition $\mathrm{Fe}^{0}$ particles to polymerization medium, the intensities of absorption band of Ppy/Fe composite are increased. Also, the synthesized with highest Fe amount; Ppy/Fe (1:2) has third characteristic absorption peak of Ppy around $600 \mathrm{~nm}$ at visible range.

The optical band gaps (Eg) of synthesized Ppy and Ppy/Fe composites were calculated with the help UV-vis absorption spectra using Tauc plot [32, 33]. The Eg values of the Ppy and Ppy/Fe at UV and visible range have been computed by extrapolation of the plot of $(\alpha h v)^{2}$ vs hv in Fig. 2(b), and the results are shown in Fig. 2(b) inset. The Eg value of Ppy is 3.31 and $2.57 \mathrm{eV}$ at UV and visible region. The Eg values of $\mathrm{Ppy} / \mathrm{Fe}$ at $\mathrm{UV}$ region changed from $3.30 \mathrm{eV}$ to $3.37 \mathrm{eV}$. Also, the Eg values at visible range have been changed from 2.57 to $2.34 \mathrm{eV}$. After the $\mathrm{Fe}^{0}$ addition, The Eg values
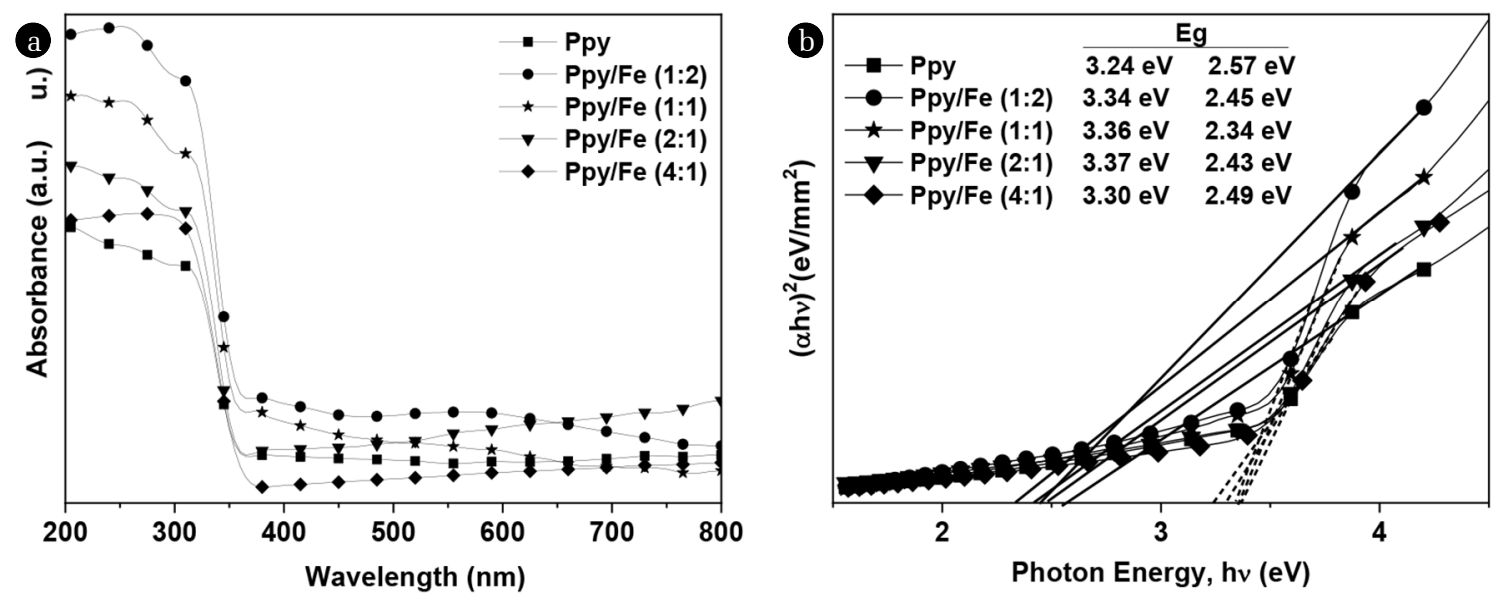

Fig. 2. (a) UV-vis diffuse reflectance spectra and (b) band gap calculation of Ppy, Ppy/Fe (4:1), Ppy/Fe (2:1), Ppy/Fe (1:1), Ppy/Fe (1:2).
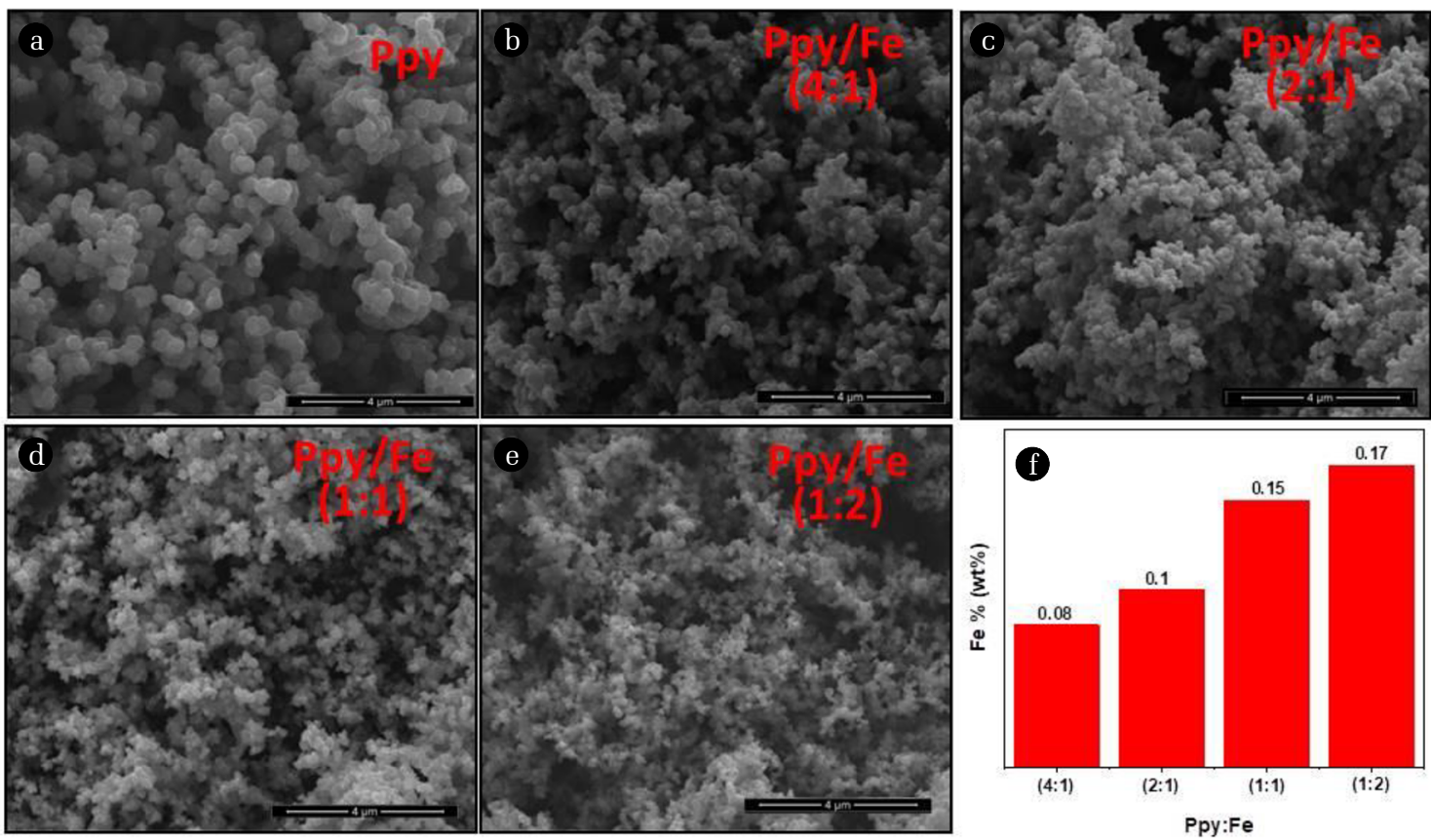

Fig. 3. SEM photograps of (a) Ppy, (b) Ppy/Fe (4:1), (c) Ppy/Fe (2:1), (d) Ppy/Fe (1:1), (e) Ppy/Fe (1:2) and (f) Fe amount (wt\%) in Ppy/Fe nanocomposite. 
of the Ppy increased in the UV region while they decreased in the visible region. The smallest Eg value belongs to Ppy/Fe (1:1) in the visible range.

The SEM images of Ppy and Ppy/Fe composites are given in Fig. 3(a)-(e). The morphology is one of the key factor for photocatalysis because of effected the photocatalytic efficiency directly [34]. All SEM images are recorded same magnification given in Fig. 3. As can be seen from SEM images, the morphology are roughly spherical in shape with sizes Fig. 3(a) and with the increase in adding $\mathrm{Fe}^{0}$ particles in the polymerization medium grain size decreases. Fe amount in Ppy/Fe composite were determined via EDX methods and the results were given in Fig. 3(f). As shown in Fig. 3(f), as the amount or $\mathrm{Fe}^{0}$ particles increased in polymerization medium, the percentage of $\mathrm{Fe}$ (wt\%) increases in the polymer composite.

The electrical conductivity of Ppy and Ppy/Fe composite were given in Fig. 4. The electrical conductivity of pure Ppy is $1.7 \times 10^{-5}$ $\mathrm{S} / \mathrm{cm}$. When Ppy combined with the $\mathrm{Fe}^{0}$ particles, the electrical conductivity is increased. The conductivity increases with increase in $\mathrm{Fe}^{0}$ contents in Ppy composite. The electrical conductivity of Ppy/Fe (1:1) is ten times higher than pure polymer. The conductivity reached its maximum value for Ppy/Fe (1:1), but it partially decreased when the amount of $\mathrm{Fe}^{0}$ in the composite was increased further. The reason for the partial decrease in conductivity at high $\mathrm{Fe}^{0}$ amount is the disruption of the homogeneous distribution of $\mathrm{Fe}^{0}$ nanoparticles in the composite and the formation of agglomeration. The conductivity of a photocatalyst depends of the availability of electrons in the conduction band. In conductive polymer, the band gap small enough to excite the valence band electrons to conduction band by absorbance of light. So if the band gap of a photocatalyst is small, the conductivity will increase. As electrical conductivity increased, the charge transfer ability rise [34]. The change in conductivity with the addition of $\mathrm{Fe}^{0}$ particles to the polymerization environment is another confirmation of composite formation and polymer-Fe interactions.

Fig. 5 indicates XRD patterns of the Ppy and Ppy/Fe composite. As shown Fig. 5, Ppy is amorphous in nature and the characteristic peak of amorphous Ppy has been obtained at $2=24.6$ [35]. For the composite, the Fe nanoparticles could not influence the crystalline structure of Ppy due. However, a very weak Fe(110) diffraction peak has been detected at a diffraction angle of about $47.16^{\circ}$ [36].

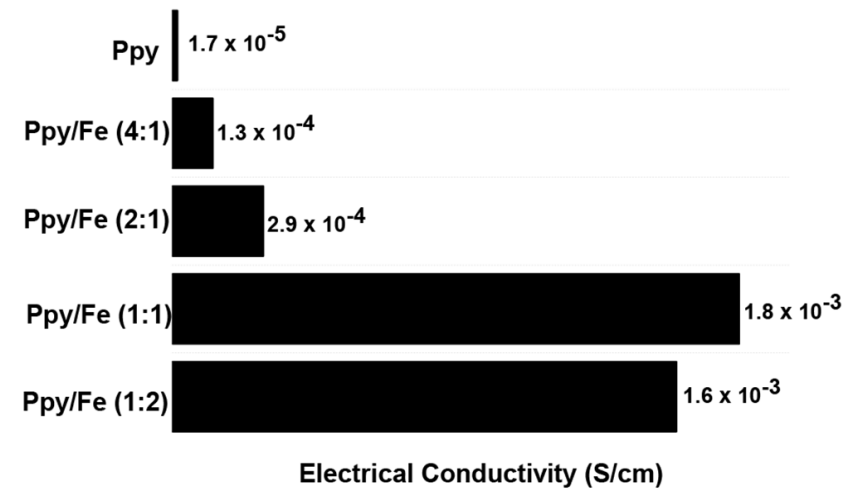

Fig. 4. Electrical conductivity of Ppy, Ppy/Fe (4:1), Ppy/Fe (2:1), Ppy/Fe (1:1), Ppy/Fe (1:2).

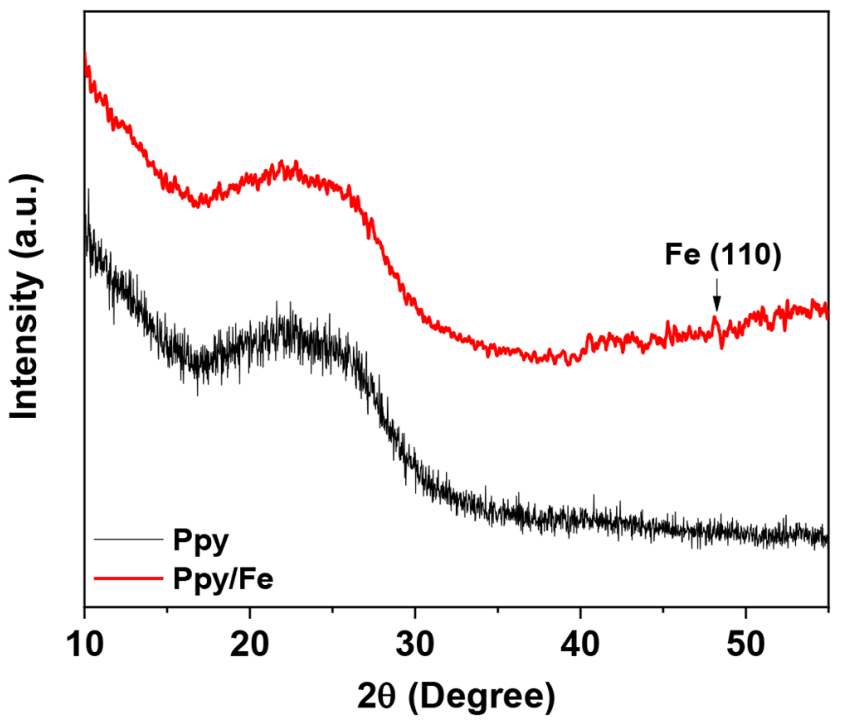

Fig. 5. XRD pattern of Ppy and Ppy/Fe.

The pHpzc of Ppy/Fe was determined by $\mathrm{pH}$ drift method. For this purpose $0.01 \mathrm{M} \mathrm{NaCl}$ solution was placed in a beaker and $\mathrm{N}_{2}$ was bubbled through the solution to stabilize the $\mathrm{pH}$ by preventing the dissolution of $\mathrm{CO}_{2}$. The $\mathrm{pH}$ was then adjusted to initial values 2, 4, 6, 8 and 10 by adding either $0.1 \mathrm{M} \mathrm{HCl}$ or $0.1 \mathrm{M}$ $\mathrm{NaOH}$ and the $0.2 \mathrm{~g}$ Ppy/Fe was added to the solution. After 48 $\mathrm{h}$, the final $\mathrm{pH}$ of $\mathrm{Ppy} / \mathrm{Fe}$ was measured and plotted the initial $\mathrm{pH}$ (Fig. S2). The $\mathrm{pH}$ at which the curve crosses the line $\mathrm{pH}$ (final) $=\mathrm{pH}$ (initial) is taken as the pHpzc of the adsorbents. The pHpzc of Ppy/Fe was determined to be 2.96. The natural $\mathrm{pH}$ value of $\mathrm{MG}$ solution was found to be 5.7. At the MG solution $\mathrm{pH}>\mathrm{pHpzc}$, the Ppy/Fe surface negatively charged and promote degradation of cationic dyes due to increased electrostatic force of attraction. At $\mathrm{pH}<\mathrm{pHpzc}$, the surface becomes positively charged, concentrations of $\mathrm{H}^{+}$were high and they compete with positively charged MG cations for vacant adsorption sites causing a decrease in dye degradation. So, MG which is cationic dye degradation by using $\mathrm{Ppy} / \mathrm{Fe}$ is favored at $\mathrm{pH}$ higher than $\mathrm{pHpzc}$.

\subsection{Photocatalytic Activities of Ppy and Ppy/Fe Nanocomposite}

The photocatalytic activity of the synthesized Ppy and Ppy/Fe composites was investigated by the degradation of MG dye under UV light illumination. Fig. 6(a) indicates the effect of Ppy:Fe ratio on photocatalytic activity of the Ppy/Fe composite materials for 60 min UV light irradiation. As shown in Fig. 6, Ppy/Fe (1:1) has higher photocatalytic activity than Ppy and other Ppy/Fe composites under UV light irradiation. According to the Fig. 6(a), optimum photocatalyst for degradation of MB dye under UV light illumination was selected Ppy/Fe (1:1). The apparent rate constant of MG photodegradation was calculated by relation following Eq. (2).

$$
\ln \left(\mathrm{C}_{\mathrm{t}} / \mathrm{C}_{0}\right)=\mathrm{k}_{\mathrm{app}} \mathrm{t}
$$

where $\mathrm{k}_{\text {app }}$ is the apparent rate constant, $\mathrm{C}_{0}$ is the initial concentration and $C_{t}$ is the concentration of dyes at the given time following pseudo-first order kinetics for different $\mathrm{Fe}$ amount in the polymer- 

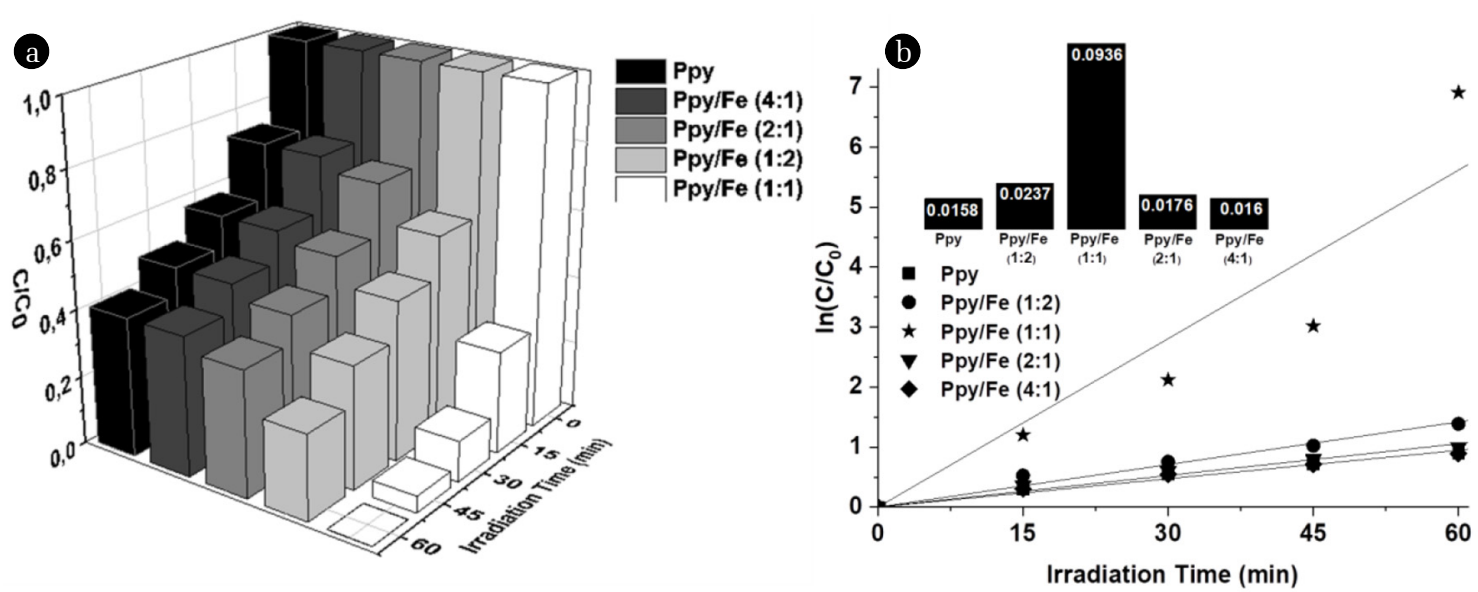

Fig. 6. (a) The effect of Ppy:Fe ratio on photocatalytic activity of the produced Ppy/Fe composite b) the relationship between $t$ and $\ln \left(\mathrm{C}_{t} / \mathrm{C}_{0}\right)$ for MG dye under UV light irradiation for different Fe amount in the polymerization medium, inset. (b) the $\mathrm{k}_{\text {aap }}$ values of Ppy and Ppy/Fe composite (dye concentration: $1.0 \times 10^{-5} \mathrm{M}$, photocatalyst amount $0.4 \mathrm{mg} / \mathrm{mL}$, light source: UV light).

ization medium (Fig. 6(b)). The $\mathrm{k}_{\mathrm{aap}}$ value of produced Ppy and Ppy/Fe composite were given inset Fig. 6(b). As can be seen from inset Fig. 6(b), the highest $k_{\text {app }}$ value, $0.0936 \mathrm{~min}^{-1}$ belongs to Ppy/Fe (1:1) polymer composite. This can be attributed of its smallest Eg value and highest electrical conductivity. When the Ppy/Fe (1:1) is exposure with UV light, an electron is jumped from valence band to conduction band. This photogenerated charge has the high transfer ability due to its high electrical conductivity. So, the Ppy/Fe (1:1) has the higher photocatalytic activity than pure Ppy and other Ppy/Fe composites. The photocatalytic activity of the catalyst can usually be improved by doping or by creating nanocomposites [9]. The high photocatalytic activity of the polymer/metal or polymer/metal oxide nanocomposites depends on the homogeneous distribution of the dopant in the polymer. With the increase in the amount of $\mathrm{Fe}^{0}$ in the $\mathrm{Ppy} / \mathrm{Fe}$ (1:2) composite, the homogeneous distribution of $\mathrm{Fe}^{0}$ is distorted and $\mathrm{Fe}^{0}$ agglomerations can be occur in the composite. As a result, a decrease in photocatalytic activity is observed.

Fig. S3 shows MG degradation in the presence of Ppy and Ppy/Fe composite under dark and UV light conditions. The MG dye stored under dark for $60 \mathrm{~min}$ for understand adsorption mechanism. The decolorization efficiency of MG dye is 14 and $8.9 \%$ with adsorption process in the presence of Ppy and Ppy/Fe composite, respectively. After 60 min UV light irradiation, the degradation efficiency of MG reached up $95 \%$ by using Ppy/Fe as photocatalysts. At the same time, pure Ppy is degraded the MG dye only $58.7 \%$ under UV light irradiation. According to Fig. S3, $\mathrm{Fe}^{0}$ particles addition to polymerization medium increased the photocatalytic activity.

The effect of photocatalysts amount on the decolorization of MG was researched under UV light irradiation a fixed 60 min irradiation time (Fig. S4). The experimental results were given in Table S1. The photocatalytic degradation rate of MG dye is increased with the increase of Ppy/Fe amount. The photocatalysts amount threshold was evaluated as $1.2 \mathrm{mg} / \mathrm{mL}$, but the photocatalytic degradation rate of MG dye for the higher photocatalyst amount loadings is related to due to irregular distribution of active sites and, presumably, agglomeration. Generally, because of the

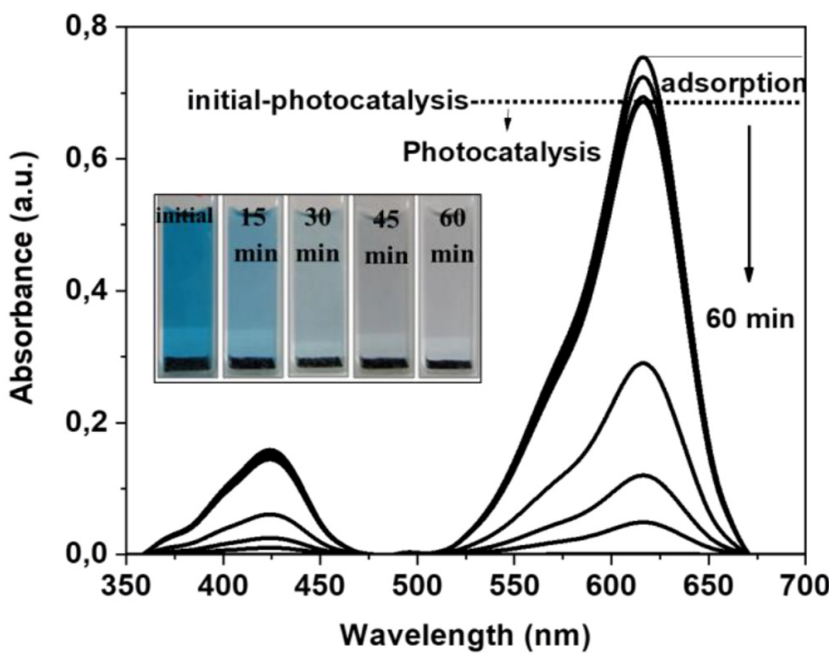

Fig. 7. UV - vis absorption spectrum of MG dye used the synthesized $\mathrm{Ppy} / \mathrm{Fe}$ as a photocatalyst for different time intervals under UV light irradiation (photocatalyst amount $1.2 \mathrm{mg} / \mathrm{mL}$; initial concentration of dyes: $1.0 \times 10^{-5} \mathrm{M}$ ).

increment in active sites, hydroxyl radical generated from illuminated photocatalyst and leads to an improvement in the rate of photocatalytic degradation. Beyond the optimum photocatalyst amount, the degradation rate is declined due to rise in the opacity of the suspension, and thus increasing the light scattering and also the infiltration depth of the photons is reduced and fewer photocatalysts could be activated. Also, the agglomeration of nanoparticles at high concentrations leads to a decline in the number of surface active sites.

Fig. 7 shows the change in absorption spectra of MG dye under UV light for different periods by using Ppy/Fe as photocatalyst. The reduction of the characteristic bands intensities of MG dye observed at 425 and $615 \mathrm{~nm}$ shows that MG has been degraded by Ppy/Fe composite under UV light irradiation in $60 \mathrm{~min}$. Also, the degradation efficiency of $\mathrm{MB}$ dye is around $61 \%$ in the presence 


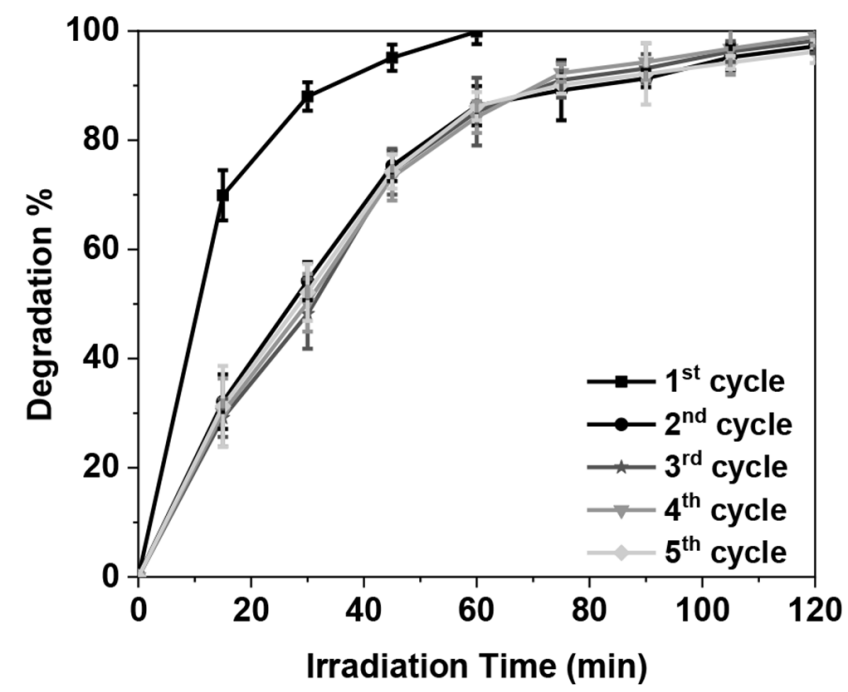

Fig. 8. The photocatalytic stability with the average of three repetitions standard deviations of Ppy/Fe.

the Ppy/Fe after only $15 \mathrm{~min}$ exposure time under UV light irradiation. After 60 min UV light illumination, MG dyes completely degraded (Fig. 7). The inset images in Fig. 7 demonstrated the decolorization of MG dye during 60 min under UV light irradiation.

To investigate the photocatalytic stability of Ppy/Fe composite, the photocatalytic decolorization experiment was recurrence up to five times. Each photocatalytic experimental results were shown the average of three repetitions \pm standard deviations and the results are given in Fig. 8. For each photocatalytic cycle, Ppy/Fe is gathered via centrifugation, and then washed with deionized water several times. After first photocatalytic usage, the photocatalytic efficiency of Ppy/Fe was decreased. MG dye degraded in 120 min under UV light irradiation in the second photocatalytic usage. As shown in Fig. 8, no apparent deactivation of $\mathrm{Ppy} / \mathrm{Fe}$ was observed after second usage under UV light irradiation for degradation of MG dye.

FTIR analyzes were carried out to confirm the photocatalytic stability of Ppy/Fe composite after fifth photocatalytic experiment. As shown, Fig. S5, the similar spectrums have obtained before and after photocatalytic degradation of MG dyes via Ppy/Fe composite under UV light irradiation. The structure of Ppy/Fe composite was not affected during photocatalytic process.

In recent studies, Yuan and co-workers [37] synthesized the Ppy $n$ hexagonal meso phases (used as soft templates) by chemical polymerization (Ppy-NS-c). The photocatalytic activity of Ppy-NS-c was investigated under UV and visible light irradiation by the degradation of phenol. Ppy-NS-c presents the highest activity for photodegradation of phenol under UV light with degradation rate of phenol about $100 \%$ after 4.5 h. In the other work; the apparent rate constant of methyl orange photodegradation is $0.0130 \mathrm{~min}^{-1}$ by $\mathrm{Ppy} / \mathrm{TiO}_{2}$ under UV light illumination [38]. The photocatalytic activity of Ag/TiO2@PPy heterostructure was studied by using methylene blue (MB) degradation under UV light irradiation. The $\mathrm{MB}$ dye completely degraded after around 100 min UV light irradiation [39]. In this study, Ppy/ Fe synthesized with a facile synthesized method and it has been performed high photocatalytic performance under UV light irradiation for the MG dye degradation.

\section{Conclusions}

In this study, Ppy/Fe composite have been synthesized via facile chemical oxidative polymerization methods in the diethylene glycol medium for the first time. The characterization of Ppy and Ppy/Fe composite were completed by using FTIR, UV-vis diffuse reflectance, SEM, EDX four probe electrical conductivity, XRD and pHpzc methods. The electrical conductivity of Ppy is increased the 10 times when $\mathrm{Fe}^{0}$ particles added to polymerization medium. The interaction between $\mathrm{Fe}^{0}$ particles and Ppy demonstrated these characterization methods. The degradation of MG dye was studied for 60 min to evaluate Ppy/Fe photocatalytic performance under UV light irradiation. The photocatalytic efficiency of Ppy/Fe composite were investigated under UV light irradiation as a function of irradiation time, ratio of Ppy and $\mathrm{Fe}^{0}$, photocatalyst amount and photocatalytic stability. MG dye completely degraded after 60 min later under UV light irradiation. The $k_{\text {app }}$ value of Ppy/Fe composite is $0.0936 \mathrm{~min}^{-1}$ for degradation of MG dye under UV light irradiation. As the results were evaluated, Ppy/Fe composite is a promising photocatalyst for degradation the textile industries wastewater by using photocatalytic wastewater treatment.

\section{Acknowledgments}

The authors are grateful for financial support by The Scientific and Technical Research Council of Turkey-2209-A - Research Project Support Programme for Undergraduate Students.

\section{Author Contributions}

B.H.T. (Ph.D) conducted experiments and wrote and revised the manuscript. H.K. (Professor) revised the manuscript. Ö.Y. (Chem.Engin.) conducted experiments

\section{References}

1. Natarajan R, Manivasagan R. Effect of operating parameters on dye wastewater treatment using Prosopis cineraria and kinetic modeling. Environ. Eng. Res. 2019;25:788-793.

2. Al-Mamun MR, Kader S, Islam MS, Khan MZH. Photocatalytic activity improvement and application of UV-TiO2 photocatalysis in textile wastewater treatment: A review. J. Environ. Chem. Eng. 2019;7:103248.

3. Balati A, Tek S, Nash K, Shipley H. Nanoarchitecture of TiO2 microspheres with expanded lattice interlayers and its heterojunction to the laser modified black TiO2 using pulsed laser ablation in liquid with improved photocatalytic performance under visible light irradiation. J. Colloid. Interface. Sci. 2019;541:234-248.

4. Balati A, Bazilio A, Shahriar A, Nash K, Shipley HJ. 
Simultaneous formation of ultra-thin MoSe2 nanosheets, Inorganic Fullerene-Like MoSe2 and MoO3 quantum dots using fast and ecofriendly Pulsed Laser Ablation in Liquid followed by microwave treatment. Mater. Sci. Semiconduc. Proces. 2019;99:68-77.

5. Singh P, Sharma K, Hasija V, et al. Systematic review on applicability of magnetic iron oxides-integrated photocatalysts for degradation of organic pollutants in water. Mater. Today Chem. 2019;14:100186-27.

6. Balati A, Wagle D, Nash KL, Shipley HJ, Heterojunction of TiO2 nanoparticle embedded into ZSM5 to 2D and 3D layered-structures of MoS2 nanosheets fabricated by pulsed laser ablation and microwave technique in deionized water: structurally enhanced photocatalytic performance. Appl. Nanosci. 2018;9:19-32.

7. Balati A, Matta A, Nash K, Shipley HJ. Heterojunction of vertically aligned MoS2 layers to Hydrogenated Black TiO2 and Rutile Based Inorganic Hollow Microspheres for the highly enhanced visible light arsenic photooxidation. Comp. Part $B$ Eng. 2020;185;107785.

8. Yar A, Haspulat B, Üstün T, et al. Electrospun TiO2/ZnO/PAN hybrid nanofiber membranes with efficient photocatalytic activity. RSC Adv. 2017;7:29806-29814.

9. Haspulat Taymaz B, Eskizeybek V, Kamis H. A novel polyaniline/NiO nanocomposite as a UV and visible-light photocatalyst for complete degradation of the model dyes and the real textile wastewater. Environ. Sci. Pollut. Res. 2020;28(6):1-19.

10. Zhang Z, Liu L, Huang H, et al. Encapsulation of CsPbBr3 perovskite quantum dots into PPy conducting polymer: Exceptional water stability and enhanced charge transport property. Appl. Surf. Sci. 2020;526:146735-146738.

11. Dinh PH, Pham TD, Trung DQ, et al. CuWO4 decorated by polypyrrole (PPy) protector/sensitizer for novel photocatalytic and stable water splitting for hydrogen generation. Int. J. Hydrog. Energy. 2020;45:21442-21449.

12. Gopal RA, Song M, Yang D, Lkhagvaa T, Chandrasekaran S, Choi D. Synthesis of hierarchically structured $\gamma-\mathrm{Fe} 2 \mathrm{O} 3-\mathrm{PPy}$ nanocomposite as effective adsorbent for cationic dye removal from wastewater. Environ. Pollut. 2020;267:115498-11.

13. Tong Y, Liu H, Dai M, Xiao L, Wu X. Metal-organic framework derived Co3O4/PPy bifunctional electrocatalysts for efficient overall water splitting. Chin. Chem. Lett. 2020;31:2295-2299.

14. Ahmad N, Sultana S, Sabir S, Khan MZ. Exploring the visible light driven photocatalysis by reduced graphene oxide supported Ppy/CdS nanocomposites for the degradation of organic pollutants. J. Photochem. Photobio. A Chem. 2020;386:112129112113.

15. Ceretta MB, Vieira Y, Wolski EA, Foletto EL, Silvestri S. Biological degradation coupled to photocatalysis by $\mathrm{ZnO} /$ polypyrrole composite for the treatment of real textile wastewater. J. Water Process. Eng. 2020;35:101230-101239.

16. Mittal H, Khanuja M. Hydrothermal in-situ synthesis of MoSe2-polypyrrole nanocomposite for efficient photocatalytic degradation of dyes under dark and visible light irradiation. Sep. Purif. Tech. 2021;254:117508-117513.

17. Madan S, Shaw R, Tiwari S, Tiwari SK. Enhancing corrosion stability and shelf life of nanoscale zero-valent iron via encapsu- lation in porous Ze-TiO2 matrix: An interface for simultaneous oxidation and adsorption of As(III). Coll. Surf. A Physicochem. Eng. Asp. 2020;607:125381-125312.

18. Sun D, Hong X, Wu K, Hui KS, Du Y, Hui KN. Simultaneous removal of ammonia and phosphate by electro-oxidation and electrocoagulation using RuO2-IrO2/Ti and microscale zero-valent iron composite electrode. Water Res. 2020;169:115239115211.

19. da Silva MTP, Villarroel-Rocha J, Toncón-Leal CF, et al. Textural and photocatalytic characteristics of iron-cobalt based nanocomposites supported on SBA-15: Synergistic effect between $\mathrm{Fe} 2+$ and $\mathrm{Fe} 0$ on photoactivity. Micropor. Mesopor. Mater. 2021;310:110582-110511.

20. Rabé K, Liu L, Nahyoon NA, et al. Fabrication of high efficiency visible light Z-scheme heterostructure photocatalyst g-C3N4/Fe0(1\%)/TiO2 and degradation of rhodamine B and antibiotics. J. Taiwan Inst. Chem. Eng. 2019;96:463-472.

21. Tanwar R, Kumar S, Mandal UK. Photocatalytic activity of $\mathrm{PANI} / \mathrm{Fe}^{0}$ doped $\mathrm{BiOCl}$ under visible light-degradation of Congo red dye. J. Photochem. Photobio. A. Chem. 2017;333:105-116.

22. Yue X, Liu Z, Zhang Q, et al. Oxidative degradation of Rhodamine $\mathrm{B}$ in aqueous solution using $\mathrm{Fe} / \mathrm{PANI}$ nanoparticles in the presence of AQS serving as an electron shuttle. Desalin. Water Treat. 2015;57:15190-15199.

23. Bhaumik M, McCrindle RI, Maity A. Enhanced adsorptive degradation of Congo red in aqueous solutions using polyaniline/ $\mathrm{Fe}^{0}$ composite nanofibers. Chem. Eng. J. 2015;260:716-729.

24. Haspulat B, Çınar Ö, Kamış H. Synthesis of Polypyrrole in Aqueous Diethylene Glycol Solution. Selcuk Uni. J. Eng. Sci. Tech. 2014;2:01-08.

25. Sen S, Gök A, Gülce H. Novel Ni/polypyrrole and Cu/polypyrrole composites prepared in the presence of different acids: Synthesis and investigation of thermal stability. J. App. Polym. Sci. 2007;106:3852-3860.

26. Haspulat B, Sarıbel M, Kamıs H. Surfactant assisted hydrothermal synthesis of $\mathrm{SnO}$ nanoparticles with enhanced photocatalytic activity. Arabian J. Chem. 2020;13:96-108.

27. Muthusamy S, Charles J, Renganathan B, Sastikumar D. In situ growth of Prussian blue nanocubes on polypyrrole nanoparticles: facile synthesis, characterization and their application as fiber optic gas sensor. J. Mater. Sci. 2018;53:15401-15417.

28. Canoluk C, Gursoy SS. Chemical modification of rose leaf with polypyrrole for the removal of $\mathrm{Pb}$ (II) and Cd (II) from aqueous solution. J. Macromol. Sci. Part A. 2017;54:782-790.

29. Kadhum ST, Alkindi GY, Albayati TM. Eco friendly adsorbents for removal of phenol from aqueous solution employing nanoparticle zero-valent iron synthesized from modified green tea bio-waste and supported on silty clay. Chin. J. Chem. Eng. 2020. (In Press)

30. Bilal S, Sohail M, Shah AHA. Synthesis and Characterization of Soluble and Thermally Stable Polypyrrole-DBSA Salts. J. Chem. Soc. Pakisthan. 2014;36:976-982.

31. Roy S, Mishra S, Yogi P, Saxena SK, Sagdeo PR, Kumar R. Synthesis of Conducting Polypyrrole-Titanium Oxide Nanocomposite: Study of Structural, Optical and Electrical Properties. J. Inorg. Organometal. Poly. Mater. 2017;27:257-263. 32. Gülce H, Eskizeybek V, Haspulat B, Sarı F, Gülce A, Avcı A. 
Preparation of a New Polyaniline/CdO Nanocomposite and Investigation of Its Photocatalytic Activity: Comparative Study under UV Light and Natural Sunlight Irradiation. Ind. Eng. Chem. Res. 2013;52:10924-10934.

33. Avcı A, Eskizeybek V, Gülce H, Haspulat B, Şahin ÖS. ZnO-TiO2 nanocomposites formed under submerged DC arc discharge: preparation, characterization and photocatalytic properties. Appl. Phys. A. 2013;116:1119-1125.

34. Karthikeyan C, Arunachalam P, Ramachandran K, Al-Mayouf AM, Karuppuchamy S. Recent advances in semiconductor metal oxides with enhanced methods for solar photocatalytic applications. J. Alloy Comp. 2020;828:154281-154215.

35. Thayyath SA, Peethambaran LD, Jayachandran N. Utilization of polypyrrole coated iron-doped titania based hydrogel for the removal of tetracycline hydrochloride from aqueous sol- utions: Adsorption and photocatalytic degradation studies. Environ. Nanotech. Monitor. Manage. 2015;4:106-117.

36. Liu R, Qiu H, Zong H, Fang C, Fabrication and Characterization of Composite Containing HCl-Doped Polyaniline and $\mathrm{Fe}$ Nanoparticles. J. Nanomater. 2012;2012:1-7.

37. Yuan X, Floresyona D, Aubert PH, et al. Photocatalytic degradation of organic pollutant with polypyrrole nanostructures under UV and visible light. App. Catal. B Environ. 2019;242:284-292.

38. Luo Q, Li X, Wang D, Wang Y, An J. Photocatalytic activity of polypyrrole/TiO2 nanocomposites under visible and UV light. J. Mater. Sci. 2010;46:1646-1654.

39. Kumar R, El-Shishtawy R, Barakat M. Synthesis and Characterization of Ag-Ag2O/TiO2@polypyrrole Heterojunction for Enhanced Photocatalytic Degradation of Methylene Blue. Catalysts 2016;6:76, 1-11. 